\title{
Dialysis: a field moving forward
}

A host of innovative developments in dialysis technologies could potentially transform the field, with benefits for patient outcomes, access to therapy and environmental sustainability.

Dialysis is a lifeline for patients with kidney failure who without a kidney transplant would otherwise die. But current dialysis modalities are far from adequate. The treatment is expensive, prohibitively so for many regions of the world; it is environmentally damaging; and outcomes for many patients remain unacceptable. Despite these issues, the field has seen very little innovation since the first iterations of dialysis machines more than 60 years ago. The reasons for this lack of progress are manyfold, but likely include vast underfunding for kidney disease research; the misconception that kidney function can be adequately 'replaced' by dialysis; and the monopoly of large dialysis companies, which have little incentive to innovate. These obstacles are by no means trivial; however, innovation in the dialysis space is beginning to increase. In this Focus issue, we highlight some of the innovative strategies currently under development.

To understand the need for innovation, the shortcomings of current dialysis modalities must be appreciated. In their Review, Jonathan Himmelfarb and colleagues provide an overview of the current dialysis landscape, describing how the global dialysis population continues to increase and how the high costs of dialysis result in inequitable access to treatment. In 2010, an estimated 2.3-7.1 million people who needed kidney replacement therapy (KRT) did not receive it ${ }^{1}$; this number is projected to increase to 9 million in 2030 $\left(\mathrm{REF}^{2}\right.$ ). Patients who do receive dialysis experience a high residual disease burden, low health-related quality of life and shortened life expectancy. In the USA, 5-year survival on dialysis is only $\sim 40 \%$, far worse than that for several solid-organ cancers ${ }^{3,4}$.

The success of haemodialysis - the most widely used form of KRT - requires a reliable vascular access; however, access-related complications are common and associated with high morbidity and mortality as well as exorbitant costs. In their Review, Jeffrey Lawson and colleagues outline the limitations of current vascular access techniques and discuss novel technologies that could potentially address these issues.

The functions of the kidney are enormously complex, acting to not only filter blood but also to maintain whole-body fluid and electrolyte homeostasis, and contributing to a host of other processes. Current modes of dialysis are unable to replicate all of these functions, in part because of their intermittent nature and inability to adequately remove various toxins and normalize electrolyte levels. The development of new, improved modes of
KRT therefore requires consideration of multiple aspects of kidney function. Progress will depend on a coordinated, multidisciplinary approach, with input from nephrologists, biophysicists, engineers, material scientists and others. To this end, this Focus issue explores the views of various stakeholders on the innovations that are needed to improve outcomes for patients on dialysis. At the forefront of this discussion should be the patients themselves. Indeed, as emphasized by Nieltje Gedney and co-authors, innovators must engage with patients (dialysors) throughout the development process to ensure that individualized solutions are available to suit their differing needs.

Also central to the process of device innovation are the regulators, who are ultimately responsible for their approval. The steps taken by regulatory and related agencies to coordinate processes between different stakeholders and jurisdictions and expedite device approval are crucial to promote innovation, particularly by small and medium-sized enterprises, and to accelerate patient access to innovative technologies.

Finally, comment pieces written by leaders in the field highlight just some of the innovative approaches that could potentially transform the lives of patients with kidney failure worldwide, including novel devices, membrane technologies, water purification systems and smart sensors for real-time patient monitoring.

The drive for innovation in dialysis is long overdue. The current momentum of the field is cause for celebration but must be maintained. Organizations such as the Kidney Health Initiative, the Kidney Innovation Accelerator and the Dutch Kidney Foundation foster innovation. However, advocacy efforts must be intensified to enlist the cooperation of other funding and governmental agencies to ensure that research in this field is adequately funded. Continued public awareness efforts are also key to raise understanding of the inadequacies and untenable nature of current dialysis technologies. Complacency is not an ethical or sustainable option.

\footnotetext{
Liyanage, T. Worldwide access to treatment for end-stage kidney disease: a systematic review. Lancet 385, 1975-1982 (2015).

2. International Society of Nephrology. ISN Global Kidney Health Atlas. 2nd edn, https://www.theisn.org/global-atlas (2019).

3. United States Renal Data System Annual Data Report 2018 : https://www.usrds.org/media/2283/2018_volume_2_esrd_in_the_ us.pdf (2018).

4. Naylor, K. L. Mortality in incident maintenance dialysis patients versus incident solid organ cancer patients: a population-based cohort. Am. J. Kidney Dis. 73, 765-776 (2019).
} 